\section{Laparoscopic Fenestration for a Giant Liver Cyst}

We report here on a case of a giant liver cyst treated by laparoscopic fenestration. A 71-year-old female patient was admitted with right abdominal tenderness and a palpable mass in the right abdomen; the symptoms had lasted for some months. Ultrasonography revealed a large liver cyst, approximately $14 \mathrm{~cm}$ in diameter. Laparoscopy was performed under general anesthesia, and a giant cyst with a smooth and slightly transparent wall (Figure 1) was found at the lower right face of the liver. Laparoscopy-guided needle aspiration (a total of $1600 \mathrm{ml}$ was aspirated) was carried out first in order to decrease the size of the cyst and to avoid leakage of cystic fluid into the abdominal cavity. The fenestration procedures started with an incision $1 \mathrm{~cm}$ right of the gallbladder. As much as possible of the large roof of the cyst was removed using laparoscopic sissors and a heat-cutting probe (Figure 2). Electric heat-probe cauteriza-

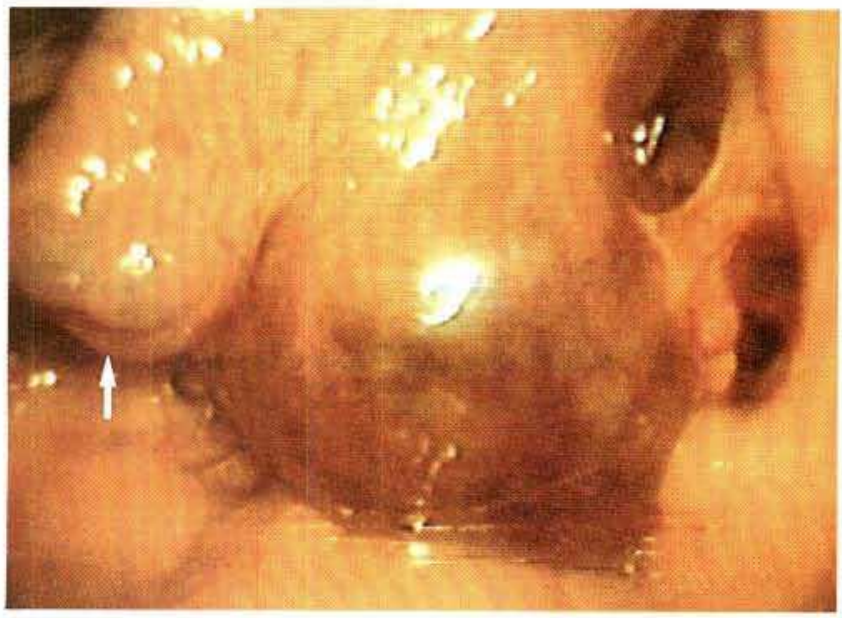

Figure 1: Laparoscopic view of a giant cyst, with a slightly transparent and smooth wall, adjacent to the inferior surface of the right liver.

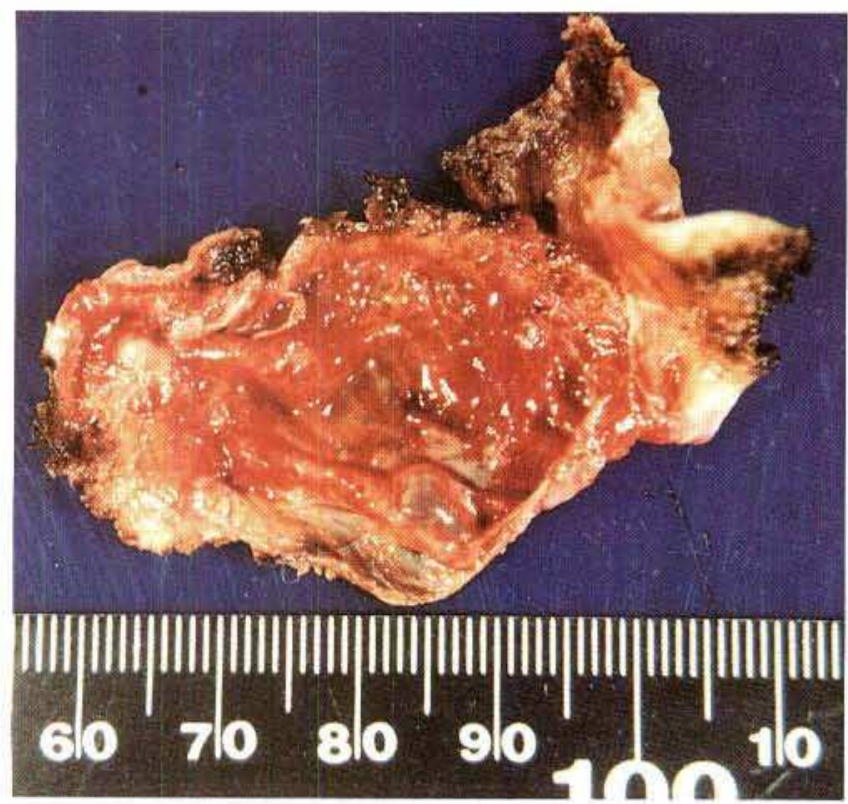

Figure 3: The resection specimen of the cyst wall, measuring about $5 \times 8 \mathrm{~cm}$ in size. tion was applied along the resection line for complete hemostasis. The inside of the cyst wall revealed whitish fibrotic changes. A silicon tube was inserted to achieve drainage. The specimen was taken out through the trocar, and it measured $5 \times 6 \mathrm{~cm}$ in size, as shown in Figure 3. Microscopically, the cyst walls were seen to be composed of either flat epithelium or to have no epithelial lining. The patient was discharged three days after laparoscopic fenestration without any morbidity. One week later, ultrasonography showed that the giant cyst had collapsed, and probably filled with omentum. Later follow-up, including ultrasonography, showed that the giant cyst was completely absent, and the patient was symptomfree after treatment.

Fenestration for giant liver cysts was first mentioned in Taiwan in 1963 (1), and the use of laparoscopic techniques has only occasionally been reported $(2-6)$. Indications for this treatment are: a) a symptomatic lesion measuring more than $5 \mathrm{~cm}$, and b) a cyst wall partially exposed from the liver surface.

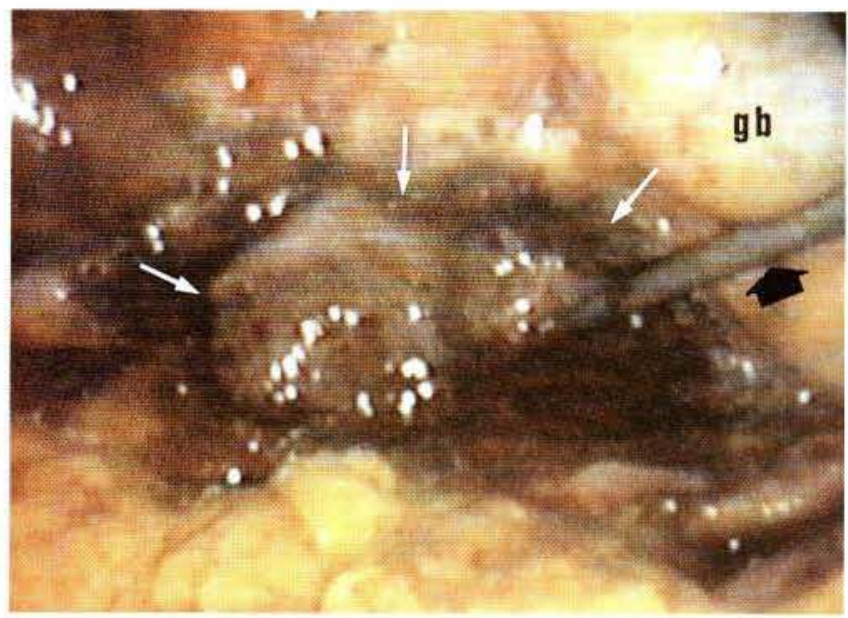

Figure 2: Laparoscopic fenestration was done to unroof the dome of this giant cyst using a heater probe and scissors (large arrow). The resected area of the cyst wall (arrows) is close to the gallbladder ( $\mathrm{gb})$.

\section{C.-G. Ker, J.-S. Chen, K.-T. Lee, P.-C. Sheen}

Division of Hepatobiliary Surgery, Kaohsiung Medical

College Hospital. Kaohsiung. Taiwan

\section{References}

1. Lin TY, Chen CC, Wang SM: Treatment of nonparasitic diseases of the liver. Ann Surg 1963; 168: $921-927$.

2. Paterson-Brown S, Garden OJ: Laser-assisted laparoscopic excision of liver cyst. Br J Surg 1991: 78 : 1047.

3. MCGiregor JR, O'Dwyer PJ: Laser-assisted laparoscopic excision of liver cyst. Br J Surg, 1992: 79: 595.

4. Moritz E: Laparoscopic fenestration of solitary giant cysts of the liver. Chirurg 1992; 63: 279-380.

5. Lange $V$, Meyer $G$, Ran $H$. Schildberg $F W$ : Minimally invasive interventions in solitary liver cysts. Chirurg 1992: 63:349-352.

6. Z'graggen K. Metzger A. Klaiber C: Symptomatic simple cyst of the liver: treatment by laparoscopic surgery. Surg Endosc 1991; 5 : $224-245$.

\section{Corresponding Author}

C.-G. Ker, M.D., Professor, Division of Hepatobiliary Surgery, Department of Surgery, Kaohsiung Medical College Hospital. Kaohsiung 800. Taiwan 